\title{
Using a fragment-based approach to identify novel chemical scaffolds targeting the dihydrofolate reductase (DHFR) from Mycobacterium tuberculosis
}

João Augusto Ribeiro ${ }^{1,2}$, Alexander Hammer ${ }^{3}$, Gerardo Andrés Libreros Zúñiga ${ }^{1,4,5}$, Sair Maximo Chavez-Pacheco ${ }^{1}$, Petros Tyrakis ${ }^{6}$, Gabriel Stephani de Oliveira ${ }^{1}$, Timothy

Kirkman $^{7}$, Jamal El Bakali ${ }^{3}$, Silvana Aparecida Rocco ${ }^{8}$, Mauricio Luís Sforça ${ }^{8}$, Roberto Parise-Filho ${ }^{9}$, Anthony G. Coyne ${ }^{3}$, Tom L Blundell ${ }^{6}$, Chris Abell ${ }^{3}$, Marcio Vinicius Bertacine Dias ${ }^{1,2,4,6,7^{*}}$.

${ }^{1}$ Institute of Biomedical Science, University of São Paulo, Brazil

${ }^{2}$ Institute of Biology, University of Campinas, Brazil

${ }^{3}$ Department of Chemistry, University of Cambridge, Lensfield Road, Cambridge, CB2 1EW, UK

${ }^{4}$ Department of Biology, IBILCE-State University of São Paulo, Brazil

${ }^{5}$ Department of Microbiology, University of Valle, Cali, Colombia

${ }^{6}$ Department of Biochemistry, University of Cambridge, Tennis Court Road, Cambridge CB2 1GA, UK

${ }^{7}$ Laboratory of Design and Synthesis of Bioactive Substances (LAPESSB), Department of Pharmacy, Faculty of Pharmaceutical Sciences, The University of São Paulo. São Paulo. Brazil.

${ }^{8}$ National Laboratory of Biosciences, Campinas, Brazil

${ }^{9}$ Department of Chemistry, University of Warwick, UK. 


\begin{abstract}
Dihydrofolate reductase (DHFR), a key enzyme involved in folate metabolism, is a widely explored target in the treatment of cancer, immune diseases, bacteria and protozoa infections. Although several antifolates have proved successful in the treatment of infectious diseases, none have been developed to combat tuberculosis, despite the essentiality of M. tuberculosis DHFR (MtDHFR). Herein, we describe an integrated fragment-based drug discovery approach to target MtDHFR that has identified hits with scaffolds not yet explored in any previous drug design campaign for this enzyme. The application of a SAR by catalog strategy of an in house library for one of the identified fragments has led to a series of molecules that bind MtDHFR with low micromolar affinities. Crystal structures of MtDHFR in complex with compounds of this series demonstrated a novel binding mode that differs from other DHFR antifolates, thus opening perspectives for the development of novel and relevant MtDHFR inhibitors.
\end{abstract}




\section{Introduction}

Dihydrofolate reductase (DHFR) is a key enzyme involved in the biosynthesis of tetrahydrofolate, an essential co-factor for the biosynthesis of purine and thymidine nucleotides and several amino acids ${ }^{1}$. DHFR is a well established target for human diseases, including cancer ${ }^{2}$, arthritis and other autoimmune diseases ${ }^{3}$, as well as for bacterial $^{4}$ and protozoa infectious diseases, such as cystitis and malaria ${ }^{5,6,7}$. However, drugs such as antifolates used for human and infectious disease treatment differ considerably, due to differences in binding sites of microorganisms and human DHFRs; therefore the antifolate drugs targeting microorganisms generally have low side effects.

Despite the importance of DHFR inhibitors for human health, there is no drug developed which targets DHFR from M. tuberculosis (MtDHFR), possibly due to the low affinity of antifolates to this protein or the impermeability of M. tuberculosis cell wall to these compounds. However, several genes of the folate pathway in M. tuberculosis have been demonstrated to be essential. These include folA, which produces $\mathrm{DHFR}^{8,9}$ and for which there is extensive literature and using different strategies that have led to a large number of potential inhibitors ${ }^{10,11,12}$. Although methotrexate (MTX) is not used therapeutically against infections, it does inhibit M. tuberculosis growth in vitro ${ }^{13} \cdot p$-Aminosalicylic acid (PAS), a second-line drug used in the treatment of tuberculosis, has been proposed to be an alternative substrate of the folate pathway and the product of the metabolism could also inhibit MtDHFR or the thymidylate synthase ${ }^{14}$.

Tuberculosis is one of the major infectious diseases and the cause of more deaths than any other bacterial species ${ }^{15}$. This disease has a high prevalence in a number of countries in Asia and Africa however this is now becoming a worldwide threat ${ }^{16}$. Various efforts have been focused on new strategies to combat tuberculosis, but only recently have two new drugs with novel action mechanisms been approved for use ${ }^{17}$. The discovery of new 
drugs against tuberculosis is particularly demanding because of the long regime therapy and the emergence of resistant strains against all used drugs. In this context, the development of new strategies to fight tuberculosis is an urgent need.

Fragment-based drug discovery (FBDD) is a successful approach that was introduced in companies such as Astex Pharmaceuticals, Abbott Laboratories and several other companies ${ }^{18}$. Although this technique is still in development, about over 40 new drugs derived from fragments have been entered in clinical trials and four of them have been approved $^{19}$. As the FBDD approach relies on the use of a small library with generally 1000 compounds, this technique has been widely applied in both the pharmaceutical industry $^{20}$ and in academia ${ }^{21,22}$. The FBDD approach involves the identification of low molecular weight compounds, with about 200-250 Da through a range of biophysical ${ }^{23}$, biochemical $^{24}$ and crystallographic methods ${ }^{25}$. Once the binding of the compounds (fragments) to the target has been validated and the binding mode elucidated, these small compounds are a very attractive starting point for the synthesis of high-affinity compounds $^{26}$. Fragments identified from a fragment screen generally have affinities between $2 \mathrm{mM}$ and $100 \mu \mathrm{M}$, and these can be quickly elaborated by organic synthesis to optimise interactions and give lead compounds that have sub-micromolar affinities for the target enzyme ${ }^{26}$. Although FBDD has been applied to several targets from $M$.

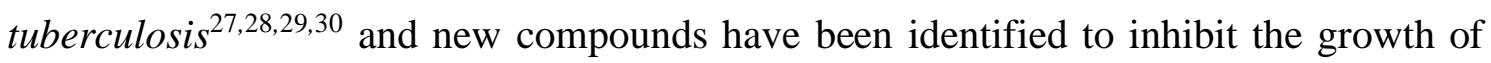
this bacterium ${ }^{29,31}$, this technique has not been applied to MtDHFR or any other DHFR.

In this work, the FBDD approach has been successfully applied using a range biophysical techniques, including Differential Scanning Fluorimetry (DSF), Isothermal Titration Calorimetry (ITC), ligand-based Nuclear Magnetic Resonance (NMR) and Crystallography to identify compounds that have completely novel scaffolds that bind to the active site of the $M t$ DHFR with $2 \mathrm{mM}$ to $80 \mu \mathrm{M}$ affinities. In addition, one of these 
compounds was used as a starting point for further elaboration where from an in house library of compounds low micromolar affinity compounds with a completely novel scaffold for a DHFR inhibitors were identified. These compounds that target DHFR do not contain the 2,4-diaminopyrimidine moiety which is found widely in DHFR inhibitors. Furthermore, $13 \mathrm{X}$-ray crystal structures of MtDHFR in complex with different molecules were solved and these will prove to be crucial in the design of new antifolates that could contribute to further tuberculosis drug discovery campaigns.

\section{Material and Methods}

\section{Protein production and preparation}

MtDHFR was produced according to the protocol established by Dias et al..$^{32}$ and that of Ribeiro et al. ${ }^{33}$ with a few modifications. Briefly, the folA gene from M. tuberculosis inserted into pET28(b) expression vector was transformed in BL21(DE3) cells and the expression was induced using $0.2 \mathrm{mM}$ IPTG at $18^{\circ} \mathrm{C}$ overnight by constant agitation at $220 \mathrm{rpm}$. The cells were resuspended in $20 \mathrm{mM}$ potassium phosphate buffer, $\mathrm{pH}$ 7.5, 50 $\mathrm{mM} \mathrm{KCl}$ (Buffer A) and disrupted by sonification. The cell debris was removed by centrifugation at 14,000 rpm and the supernatant was loaded onto an IMAC column (GE Healthcare) charged with nickel. The protein was eluted using buffer A plus $500 \mathrm{mM}$ of imidazole (buffer B), using a linear gradient of buffer B to increase the concentration of imidazole on an ÄKTA purifier system (GE Healthcare). The protein was concentrated and further purified using an S200 16/60 gel filtration chromatography column (GE Healthcare) previously equilibrated with buffer A. The protein was concentrated to at least $10 \mathrm{mg} / \mathrm{mL}^{-1}$ and stored at $-80^{\circ} \mathrm{C}$.

\section{Differential Scanning Fluorimetry (DSF)}


DSF assays were performed in a PCR iCycler (BioRad) coupled to an iQ5 Multicolor Real-Time PCR Detection System (Bio-RAD). 96 well plates were used with each well containing a total volume of $100 \mu \mathrm{L}$ consisting of $95 \%(\mathrm{v} / \mathrm{v})$ buffer A, 5\% (v/v) DMSO, 2.5x SYPRO Orange ${ }^{\circledR}$ dye (Invitrogen) and $1 \mathrm{mM}$ NADPH (Sigma-Aldrich). The final concentration of MtDHFR in each well was $3 \mu \mathrm{M}$. 80 wells of the plate were used to screen different fragments, which had a concentration of $5 \mathrm{mM}, 8$ wells were used as positive controls ( $5 \mathrm{mM}$ of trimethoprim) and 8 wells were used only in the presence of 5\% DMSO as "negative control". 1,250 fragment-like compounds from the Maybridge RO3 library were tested. For the assays, the plates were heated from $25^{\circ} \mathrm{C}$ to $75^{\circ} \mathrm{C}$ at increments of $0.5^{\circ} \mathrm{C}$ per minute. The fluorescence intensity of SYPRO Orange ${ }^{\circledR}$ dye was monitored, with excitation-emission wavelengths of $490 / 575 \mathrm{~nm}$, as a function of temperature. The $\Delta \mathrm{Tm}$ was calculated from the difference of the average values of all "negative controls" (reference) and that of the protein in the presence of fragments. A positive hit was defined as a compound that increased the melting temperature by $\geq 0.5^{\circ} \mathrm{C}$ compared to that of the reference compound.

\section{STD-NMR Assays}

For NMR experiments, the fragments were initially dissolved using DMSO- $d_{6}$ and further diluted using $\mathrm{D}_{2} \mathrm{O}$ at a final concentration of $500 \mu \mathrm{M}$ and the protein in buffer $\mathrm{A}$ was diluted to $5 \mu \mathrm{M}$. The NMR spectra were obtained using an Agilent DD2 $500 \mathrm{MHz}$ spectrometer equipped with a triple-resonance probe at $298 \mathrm{~K}$. The 1D STD-NMR spectra were obtained by the subtraction of saturated spectra (on resonance) from the reference spectra (off-resonance) after identical processing and phasing. The subtraction of onresonance spectra from off-resonance spectra was performed automatically by phase cycling using the dpfgse_satzfer pulse sequence implemented in the VNMRJ software (Agilent). The 1D STD-NMR spectrum was acquired using 8192 scans with a selective 
irradiation frequency of protein at $-0.5 \mathrm{ppm}$ for on-resonance and at $30 \mathrm{ppm}$ for offresonance. 40 Gauss-shaped pulses of $50 \mathrm{~ms}$ separated by a $1 \mathrm{~ms}$ of delay were applied to the protein. The total length of the saturation train was $2.04 \mathrm{~s} . \mathrm{A} \mathrm{T}_{2}$ filter was applied to eliminating all protein background. The off-resonance spectrum was used as a reference spectrum and acquired with 4096 scans keeping all the other parameters equal to the 1D STD-NMR spectrum. For the group epitope mapping analysis, the STD enhancements were determined by the integrals of individual protons of the ligands in the 1D STD_NMR spectrum divided by the integral of the same signals at the reference spectrum. The strongest STD enhancements in each spectrum are normalized and assigned to $100 \%$.

\section{Isothermal Titration Calorimetry (ITC)}

ITC assays were performed on a MicroCal VP-ITC 2000 or iTC200 (Malvern). For both, cell and syringe solutions contained 95\% (v/v) buffer A, $1 \mathrm{mM} \mathrm{NADPH}$ and 5\% (v/v) DMSO. The cell solution contained between 30 and $50 \mu \mathrm{M}$ of MtDHFR for experiments performed using VP-ITC 2000 or between 100 to $160 \mu \mathrm{M}$ for experiments using an iTC200. All tested compounds were dissolved at a concentration ranging from 2 to 10 $\mathrm{mM}$ in buffer $\mathrm{A}$ in the presence of in $5 \%$ of DMSO and $1 \mathrm{mM}$ of NADPH to avoid the buffer mismatch. All solutions were degassed prior to the experiments and the temperature used in the cell was $26^{\circ} \mathrm{C}$. The titration constituted of 20 to 25 injections using different volumes for VP-ITC (first injection of $3 \mu \mathrm{L}$ followed by 24 injections of $10 \mu \mathrm{L}$ ) and iTC200 (first injection of $0.2 \mu \mathrm{L}$ followed by 19 injections of $2.2 \mu \mathrm{L}$ ). The heats of dilutions were subtracted from the titration data prior to curve fitting. Most of the obtained curves were fitted by nonlinear least-squares regression using the noninteracting one-site model using Origin 7.0 (Microcal).

\section{Crystallography and structure analysis}


The crystallisation of MtDHFR was performed by the vapor diffusion method using a hanging-drop strategy and the protocol described by Ribeiro et al. ${ }^{33}$ Briefly, $1 \mu \mathrm{L}$ of MtDHFR at $10 \mathrm{mg} / \mathrm{mL}$, previously incubated with $10 \mathrm{mM}$ of $\mathrm{NADPH}$, was mixed in a coverslip with $1 \mu \mathrm{L}$ of a crystallisation condition constituted by $1.6 \mathrm{M}$ ammonium sulfate,

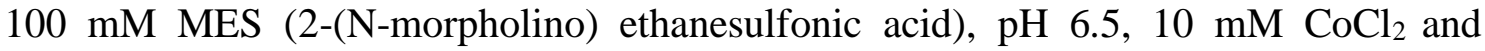
inverted down against the $300 \mu \mathrm{L}$ of crystallisation solution added in the wells of Linbro plate (Hampton Research). Bipyramidal crystals of MtDHFR appeared after 3-4 days at a temperature of $18{ }^{\circ} \mathrm{C}$. Complexes of the holoenzyme (MtDHFR:NADPH) with fragments and compounds were obtained by co-crystallisation or soaking as described in Table 1. For co-crystallisation, MtDHFR was prior incubated for about 30 minutes on ice in the presence of 20 to $60 \mathrm{mM}$ of different fragments. For soaking experiments, about 50 to $100 \mathrm{mM}$ of a compound solution was added to a drop containing crystals of holoenzyme for 10 minutes to 20 hours, prior to crystal harvesting and freezing, depending on the damage to the crystal caused by the compound.

X-ray data collection of MtDHFR in complex with different fragments and compounds was achieved using different synchrotron sources, including the Laboratório Nacional de Luz Síncrotron (Brazil) and Diamond as described in Table 1. The data processing was performed using $\mathrm{XDS}^{34}$ and scaled by Aimless ${ }^{35}$ from the CCP4 suite $^{36}$. The structures were solved by molecular replacement through the program using Phaser $\mathrm{MR}^{37}$ using the PDB entry $1 D F 7^{38}$ included in the Phenix crystallographic suite ${ }^{39}$. The crystallographic refinement was performed by the program Phenix.Refine ${ }^{40}$ followed by manual inspection using $\mathrm{COOT}^{41}$. The stereochemical quality of the models was checked using the program MolProbilty ${ }^{42}$ and the figures were prepared using PyMol (The PyMOL Molecular Graphics System, Version 2.0 Schrödinger, LLC).

\section{Results and discussion}




\section{Screening by DSF and validation by 1D STD-NMR}

MtDHFR was screened against a library of 1250 compounds selected following the rule of three ${ }^{43}$ using a fluorescence-based thermal shift method in the presence of $1 \mathrm{mM}$ of NADPH. Trimethoprim (TMP), which gave a $\Delta \mathrm{Tm}$ of $+10^{\circ} \mathrm{C}$ (Supplementary Figure 1) was used as a positive control. However, early in the screening process, it was observed that $\mathrm{T}_{\mathrm{m}}$ of negative controls varied slightly from well to well making it difficult to determine the threshold for selecting fragments. Hence, to define a threshold, 105 observations of $\mathrm{T}_{\mathrm{m}}$ of MtDHFR:NADPH in 5\% of DMSO were collected as "negative controls" and in order to verify whether these data were describing a normal distribution, two goodness-of-fit tests (Kolmogorov-Smirnov and Chi-Squared tests) were performed. Both of the tests suggested that these data are normally distributed, with a mean $T_{m}$ of $53.2^{\circ} \mathrm{C}$ and standard deviation $(\sigma)$ of $0.7^{\circ} \mathrm{C}$, up to a significance level of $\alpha=0.02$. Using this information, a 99\% confidence interval was constructed, giving a range of temperatures between 53.0 and $53.3{ }^{\circ} \mathrm{C}$, within which $99 \%$ of the mean $\mathrm{T}_{\mathrm{m}}$ values of the negative controls should lie. From this analysis, those fragments that produced flat, markedly-irregular melting curves were excluded since the $\Delta \mathrm{T}_{\mathrm{m}}$ could not be calculated. Positive hits were then defined as fragments that gave a $\Delta \mathrm{Tm}$ of $\geq 0.5^{\circ} \mathrm{C}$ above $53.4^{\circ} \mathrm{C}$. About 37 statistically positive hits (Supplementary figure 2), which represent about $2.8 \%$ of the library with significant chemical diversity, were identified when screened at a fragment concentration of $10 \mathrm{mM}$. This hit rate is similar to that obtained in several other FBDD campaigns ${ }^{27,44,24}$. The remaining molecules of the library did not significantly stabilise the MtDHFR or displayed a negative $\Delta \mathrm{T}_{\mathrm{m}}$, indicating a stabilisation of the unfolded state of MtDHFR, consequently, these molecules were also not considered for further analysis. 
A large number of identified compounds shared similar features, including a carboxylic acid at the meta position of a benzene ring, which is generally linked by a single bond to another comprising two heterocyclic rings (Figure 1). Interestingly, it has been reported that compounds with stilbenoid, deoxybenzoin and chalcone moieties, which have similar scaffolds to some of our compounds, also showed affinity to E. coli DHFR and had an uncompetitive mechanism of inhibition ${ }^{45}$ (Supplementary figure 2).

From 37 molecules that stabilised MtDHFR above than $0.5^{\circ} \mathrm{C}$ in the DSF assay, we rescreened 30 fragments (Supplementary Figure 2) using 1D STD-NMR and confirmed 21 fragments molecules (Supplementary Figure 3) that interact with the protein, which represents about $70 \%$ of the molecules (Figure 1) indicating a strong accuracy of statistic method applied for the selection of the fragments. 


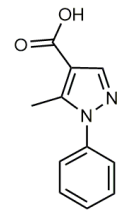

5-methyl-1-phenyl-1H-pyrazole-4-carboxylic acid

$$
1
$$

$\Delta \mathrm{Tm}=1.2^{\circ} \mathrm{C}$

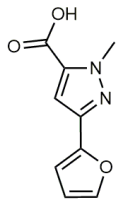

3-(furan-2-yl)-1-methyl-1H-pyrazole-5-carboxylic acid 2 $\Delta \mathrm{Tm}=1.5^{\circ} \mathrm{C}$

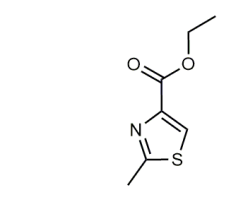

ethyl 2-methylthiazole-4-carboxylate

3

$\Delta \mathrm{Tm}=1.9^{\circ} \mathrm{C}$

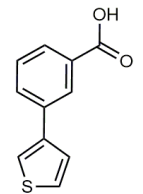

7

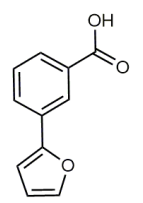

3-(furan-2-yl)benzoic acid

8

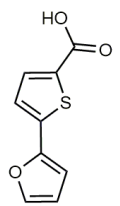

2-(oxazol-5-yl)aniline

5

$\Delta \mathrm{Tm}=0.7^{\circ} \mathrm{C}$

$\Delta \mathrm{Tm}=1.3^{\circ} \mathrm{C}$ 5-(furan-2-yl)thiophene-2-carboxylic acid

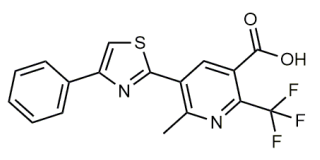

6-methyl-5-(4-phenylthiazol-2-yl)-2(trifluoromethyl)nicotinic acid

10

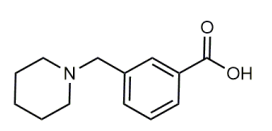

3-(piperidin-1-ylmethyl)benzoic acid 11 $\Delta \mathrm{Tm}=0.6^{\circ} \mathrm{C}$<smiles>COC(=O)Cc1cccs1</smiles>

methyl 2-(thiophen-2 4 $\Delta \mathrm{Tm}=0.5^{\circ} \mathrm{C}$
9

$\Delta \mathrm{Tm}=1.2^{\circ} \mathrm{C}$

$\Delta \mathrm{Tm}=1.7^{\circ} \mathrm{C}$

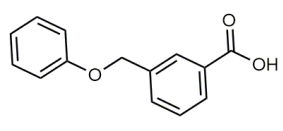

3-(phenoxymethyl)benzoic acid

14

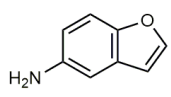

benzofuran-5-amine

15

$\Delta \mathrm{Tm}=0.7^{\circ} \mathrm{C}$

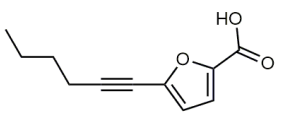

$\mathrm{Tm}=1.2^{\circ} \mathrm{C}$

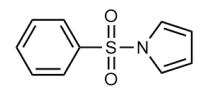

1-(phenylsulfonyl)-1 1 -pyrrole

18
19

$\Delta \mathrm{Tm}=1.7^{\circ} \mathrm{C}$

5-(hex-1-yn-1-yl)furan-2-carboxylic acid

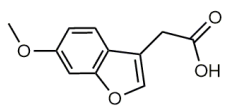

21

2-(6-methoxybenzofuran-3-yl)acetic acid

$\Delta \mathrm{Tm}=0.6^{\circ} \mathrm{C}$

$\Delta \mathrm{Tm}=0.7^{\circ} \mathrm{C}$ $\Delta \mathrm{Tm}=1.2^{\circ} \mathrm{C}$

$\Delta \mathrm{Tm}=0.7^{\circ} \mathrm{C}$

Figure 1. Fragments validated by STD-NMR. The $\Delta \mathrm{Tm}$ is included below each structure

Although 1D STD-NMR is routinely used to rescreen the hits identified by DSF, we also used this technique to identify epitopes from the fragments that are essential for the 
interaction with the target ${ }^{46}$. The identification of epitopes is possible because of the proximity of the functional groups of the side chain of amino acid residues of the target, and the degree to which this occurs is dependent on the time the ligand spends in contact with the protein. ${ }^{47}$ 1D NMR spectra of solutions containing the fragments and the holoenzyme were measured to discriminate the signals. The fragment interactions with the target were confirmed by the proton signals in the difference spectra (on-resonance off-resonance). The data of difference were normalised and the signal/noise rate was calculate and a threshold of $\geq 5$ for this relation. In addition, we have attributed to the signal of proton as the more intense for STD enhancements and consequently, we considered it as $100 \%$ and they have been used as a reference for the calculation of the intensity percentage for the other protons of the ligand. Proton signals were satisfactorily confirmed for the 21 fragments and the corresponding protons attributed in the spectra are identified by numbers in the structure. The remaining signals in the reference spectra belongs mainly to the coenzyme NADPH (Supplementary Figure 3). An interesting observation obtained by the 1D STD-NMR signal was that the benzoic acid for most of the fragments should have close contacts with the protein since the STD enhancement for this group is nearly $100 \%$. Consequently, this region should bind tightly to the target and have a key role in the interaction. In addition, it is observed that most of the compounds that have this benzoic acid moiety are linked to other heterocycles, for example, thiophene (7 and 13), furan (8), carbazole (9) and non-aromatic heterocycles, such as piperidine (11 and 12). The fragments 11 and 12 are similar molecules and they differ only in the position of the carboxylic acid at the benzene ring, which for $\mathbf{1 1}$ is at meta position and in $\mathbf{1 3}$ is para. As observed for most of the fragments with benzoic acid, $\mathbf{1 1}$ has an intense signal of protons and consequently, the meta configuration of the carboxylic acid attached to the benzene ring could be a key factor for the strong interaction. The crucial role of the 
benzoic acid for the interaction suggested that this group could perform an ionic interaction with one of the arginines within the active site of MtDHFR, such as Arg60, mimicking the interaction of glutamate moieties of dihydrofolate. The meta position of the carboxylic acid could contribute to orientate this group towards the positive charges of the active-site cavity. In addition, the importance of the carboxylic acid attached to a ring could also be observed for compounds $1,2,6$ and 10, where these instead of having benzene ring, have the carboxylic acid attached to a heterocycle such as a pyrazole, thiophene, and pyridine so that a maximum resonance could be observed for carbons of the ring. In addition, the 1D STD-NMR spectra also suggest the importance of different rings linked since the transference of resonance is highly significant. Moreover, there is a clear preference of aromatic rings, including both benzene or heterocycles, including pyrazole (1), thiazole (3), oxazole (5) and pyrrole (18). Interestingly, compound 16, which has the benzoic acid fused with a dioxepine, but the carboxylic acid attached to the benzene ring through a 4-oxo-butyl linker could have a key role in the interaction with the protein.

In addition to the fragments identified above, a number of other structures were confirmed to interact with MtDHFR. For example, another group of fragments comprises of small compounds with neither linked rings nor carboxylic acid $(\mathbf{3}, \mathbf{4}, \mathbf{1 5}, \mathbf{1 7})$. Although two fragments (5 and 18) have linked rings, they do not have carboxylic acid groups.

\section{Calorimetric analysis of fragments selected as hits for DHFR.}

The 21 fragments that were confirmed to bind to MtDHFR through DSF and 1D STDNMR, were further characterised by ITC (supplementary table 1 and supplementary Figure 4). For 5 compounds, the thermodynamic parameters were not determined due to their low solubility or there being not enough heat released in the ITC experiment conditions. For 8 molecules the affinity was too low to estimate the LE (ligand efficiency) 
or had a too-high entropic penalty, which strongly affects the $\Delta \mathrm{G}$ (Supplementary Table 1). Table 1 shows the affinity and LE for the 8 fragments. In addition, except for the titration with $\mathbf{1}$, all thermograms were fitted using a unique site model, indicating that the MtDHFR active site is occupied only by one ligand molecule (Supplementary figure 4). Interestingly, the thermogram curve for $\mathbf{1}$ indicated a sequential site model, suggesting that this molecule might bind in two distinct regions of the active site. $\mathbf{9}$ and $\mathbf{1 0}$ were the fragments that have the highest affinity among the tested molecules, with 95 and $98 \mu \mathrm{M}$, respectively, although 9 also has the highest LE, 0.34 . 
Table 1. Calorimetric analysis of fragments against MtDHFR

\begin{tabular}{|c|c|c|c|}
\hline & & \multicolumn{2}{c}{ Kd (mM) } \\
\hline
\end{tabular}




\section{Structural basis of the interaction of MtDHFR with selected fragments}

Once the fragments that interact with MtDHFR had been characterised biophysically, the next step was to define the structure of the fragments with MtDHFR in order to examine their interactions with the proteins. A total of 9 crystal structures were obtained with a resolution ranging from 2.5 to $1.7 \AA$ for MtDHFR in complex with different fragments (Supplementary Table 2). Although the binding affinity for fragments $\mathbf{3}$ and $\mathbf{1 6}$ were not able to be measured, their interaction modes with MtDHFR, despite the low occupancy of these molecules was obtained. The structures obtained for most complexes of MtDHFR:NADPH with fragments was through co-crystallisation, in which the formation of the crystal was not inhibited by the high concentration of compounds (about 15 to 40 $\mathrm{mM}$ ). The complex of MtDHFR:NADPH and $\mathbf{1 0}$ was obtained by soaking in the crystallisation condition described by Dias et al., $2014^{32}$. The crystals of MtDHFR:NADPH in complex with different fragments, except the complex of MtDHFR:NADPH with 10, belong to space group P $2{ }_{1} 2_{1} 2_{1}$ with 2 molecules in the asymmetric unit. For most of the complexes, we observed both protomer active sites occupied. Supplementary Table 2 shows the data processing, refinement and quality of the stereochemistry of the structures of the complex with fragments. All structures of MtDHFR:NADPH in complex with fragments have a closed conformation with the NADPH engaged in the active site as described previously ${ }^{32,38}$. In addition, it was observed that the protein did not undergo significant conformational changes, even in the residues of the dihydrofolate binding pocket (Supplementary Figure 5). To facilitate the description of fragment interaction with the protein, we distinguished four different subregions of the active site groove of MtDHFR:NADPH (Figure 2): (1) active site entrance, with a positive charge, (2) central region of the active site or PABA binding site, a slightly apolar region, (3) dipyrimidine binding site, with strong negative charges; and (4) the 
glycerol binding site, a specific region found in MtDHFR, but not observed in human DHFR (hDHFR) $)^{38}$.

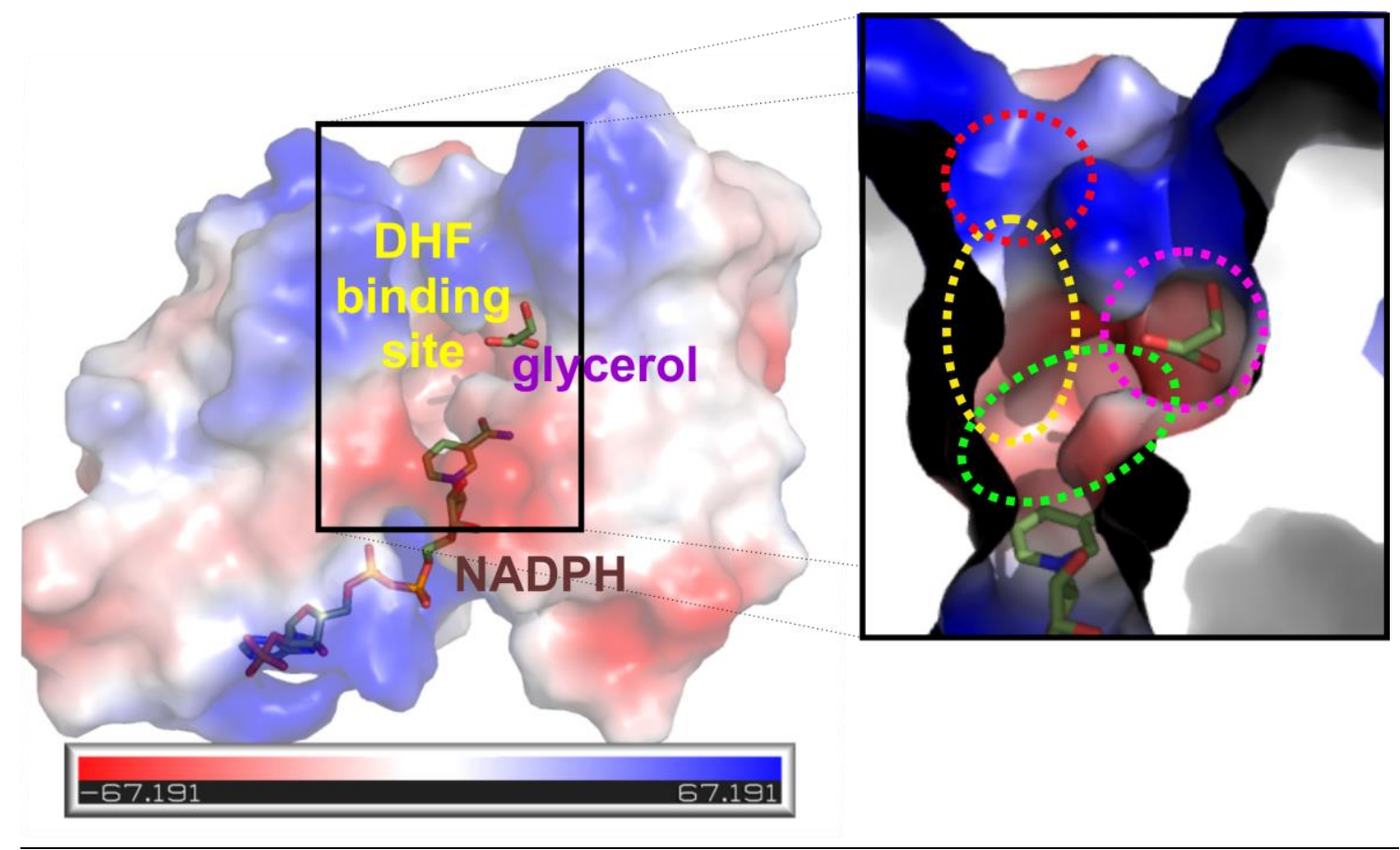

Figure 2. Active site groove of MtDHFR and subregions. The figure shows the DHF and glycerol binding cavities. In the inset, we have specified 4 subregions of the active site. 1 (in red) is a positively charged region that binds the glutamate moiety of DHF; 2 (in yellow), the central region, where the PABA moiety sits, that is apolar, 3 (in green), where the positive dipyrimidine ring of DHF stacking with nicotinamide moiety of NADPH and finally 4 (in magenta), the glycerol binding site, an exclusive pocket of MtDHFR in comparison to human DHFR.

All the structures of MtDHFR:NADPH in complex with fragments show electron density for fragments in three regions of MtDHFR:NADPH, which includes the entrance (1), PABA binding site (2) and glycerol binding site (4); surprisingly we did not observe any fragment completely occupying the dipyrimidine binding site (3), which is one of the 
most explored regions of DHFR in the development of new inhibitors. Most of the compounds that have the carboxylic acid linked to a phenyl or a heterocyclic ring indicate strong interaction with the active site entrance (region 1), where the carboxylic acid is interacting through ionic interaction with Arg60. The interactions of the compounds in that region explain and corroborate our results from 1D STD-NMR, which indicates a $100 \%$ proton transference of this region and the high enthalpic contribution for the binding, characterising a strong polar interaction. The importance of a second ring (phenyl or heterocyclic) could also be confirmed since in most cases these rings exhibit $\pi$-interactions with Phe31 on the PABA binding site (sub-region 2), as well as interactions with other residues, such as Leu50 and Ile94 (Figure 3). These interactions also confirm the results of ITC indicating high entropic contributions for the interaction of fragments (Supplementary Table 1). In addition, because of the proximity of the second ring of these compounds with the nicotinamide group of NADPH, we can also observe an edge- $\pi$ interaction with this group and then slightly reaching the subregion 3.

Fragment 2 was unique in that it did not interact with Arg60 but rather is hydrogenbonded to the side chain of Gln28 even having the carboxylic acid in meta position while maintaining the interaction with Phe31 through its furan group as do the other fragments from this group. Interestingly, fragment $\mathbf{1}$ was unique in occupying the glycerol-binding site (4), while its carboxylic acid makes a hydrogen bond with the side chain of Gln28. Since the glycerol-binding site is a feature observed in MtDHFR in contrast to hDHFR, this fragment could be an excellent starting point to obtain specific drug leads against tuberculosis. As expected, an electron density for fragment $\mathbf{1}$ was also observed in the PABA binding site (2), corroborating the results of ITC that indicated that this molecule has a sequential binding mode. However, as the average B-factor for this second molecule 
is higher than the first one, probably the binding just occurs after the complete occupation of the first site.

Although there is a structural similarity of 6-8 to $\mathbf{1}$ and $\mathbf{2}$, we were not able to obtain the structures of these fragments even though they have a carboxylic acid group. As the affinities of $\mathbf{2 , 5 - 8}$ are low, we suggest that a higher affinity interaction of this fragment scaffold would be necessary for the association of "meta-position" in a five-membered ring with a phenyl ring, a characteristic observed only for $\mathbf{1}$. Interestingly $\mathbf{1}$ and $\mathbf{2}$ are also very similar compounds, but the binding modes of these two molecules are quite different, indicating that the phenyl and furan groups, as well as the position of the methyl group attached to the pyrazole ring, might have a strong influence on the orientation of these compounds in the active site of the enzyme.

Fragments $\mathbf{3}$ and $\mathbf{1 7}$ were the only ligands in the series that do not have a carboxylic acid for which we were able to obtain the crystal structures; however, we could observe the binding mode in only one protomer of the asymmetric unit for both compounds and we were not able to obtain the affinity of these compounds by ITC. These two molecules have distinct structures since $\mathbf{3}$ is a thiazole ethyl ester and $\mathbf{1 7}$ is a trifluoromethylphenyl diamine. Interestingly, both molecules bind in the PABA binding site (3), despite their different orientations and make only non-polar contacts with the active site residues.

Fragment $\mathbf{1 0}$ is the largest for which we have obtained the crystal structure complex with MtDHFR:NADPH. Although its thiazole ring is binding in the same position of the PABA moiety of MTX, the phenyl ring reaches a deeper point in the active site of MtDHFR and sits close to the pyrimidine moiety of MTX stabilising several non-polar contacts with this active site region (Supplementary Figure 6). 
The chemical structures of the fragments $\mathbf{1 3}$ and $\mathbf{1 4}$ are similar and they differ in a second ring in which 13 and 14 have a thiophenyl-thiomethyl and a phenoxymethyl and they bind in the similar position at the active site of MtDHFR (although the twist caused by the sulfur atom in contrast to the oxygen in the linker between the two rings). The similar pose of these fragments in the active site of MtDHFR was expected since their affinities (Kd) were also similar, 0.752 and $0.502 \mathrm{mM}$ for 13 and 14, respectively. Interestingly, although these compounds are smaller than 10, the thiophene and the phenyl ring sits in the same position of the phenyl ring as compound $\mathbf{1 0}$ and the linker between the two rings adopts the position of the PABA moiety of MTX (Figure not shown).

The role of the carboxylic acid in the meta position could be further validated only by the structure of MtDHFR:NADPH in complex with 11. Even though we performed several attempts to obtain the structure for the complex with 12, crystals were not forthcoming. Although these two compounds have planar piperidine rings, $\mathbf{1 1}$ is able to perform the ionic interaction with Arg60 through its "meta" carboxylic acid. The variation in the position of the carboxylic acid attached to the phenyl rings causes a three-fold difference of affinity between the two compounds.

Finally, $\mathbf{1 6}$ is the only compound to have a benzodioxepinyl group and an oxobutanoic acid attached to position 7 of the benzyl group. Although the restraints caused by the fusion of the benzo and dioxepinyl rings on the flexibility of the oxobutanoic acid moiety, this fragment also interacts with the Arg60 through the carboxylic acid. However, the benzodioxepin moiety does not superpose well with the position of the other fragments with different linkers between the two rings and instead, only the dioxepinyl ring sits in the same binding site as the phenyl group of compound $\mathbf{1 0 .}$ 

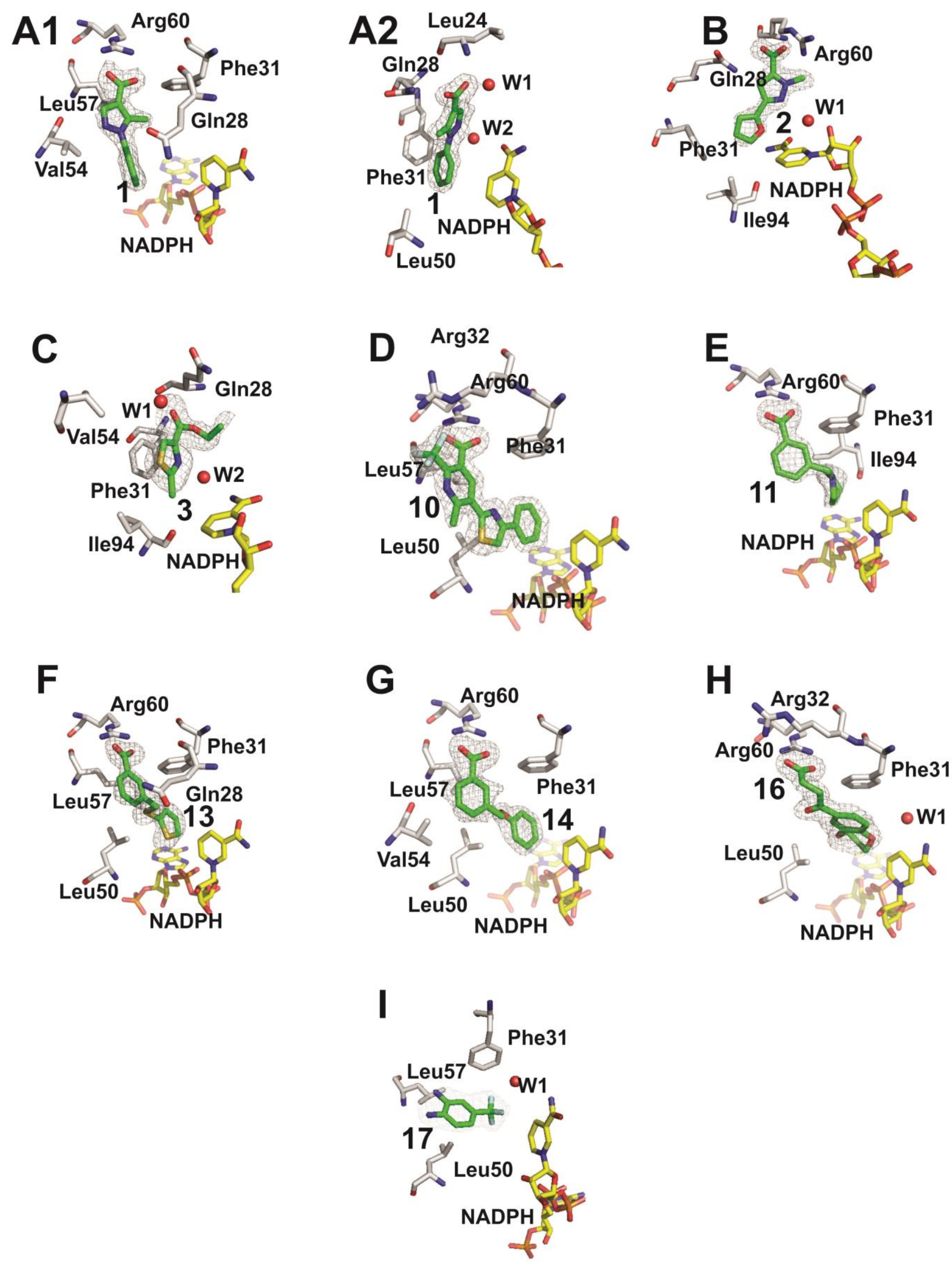
Figure 3. Crystal structures and binding modes of nine fragments that bind to MtDHFR:NADPH. (A) 1. Fragment 1 binds in two different pockets of MtDHFR (A1 and A2) (PDB entry 6VS5); (B) Fragment 2 (PDB entry 6VS6); (C) Fragment 3 (PDB entry 6VS8); (D) Fragment 10 (PDB entry 6VVB); (E) Fragment 11 (PDB entry 6VS9); (F) Fragment 13 (PDB entry 6VSD); (G) Fragment 14 (PDB entry 6VSE) ; (H) Fragment 16 (PDB entry 6VSF); (I) Fragment 17 (PDB entry 6VSG). The electron density contorns is from a 2 fo-fc map with $1 \sigma$. W1 are water molecules on the active site cavity.

\section{Applying SAR by catalog using Fragment 1 to obtain new leads with a low- micromolar affinity against MtDHFR.}

Fragment 1 binds to the glycerol pocket of MtDHFR with a $\mathrm{K}_{\mathrm{D}}$ of $0.640 \mathrm{mM}$ ( $\mathrm{LE}=0.28$ ) and it was selected for further elaboration and optimisation. Since the aryl rings of the compound play an important role in the affinity to MtDHFR, it was decided that both rings should be maintained during any further optimisation. Based on fragment 1, a campaign of SAR was explored using an in-house library (Supplementary methods). Initially, DSF was used to identify compounds that had a higher $\Delta \mathrm{T}_{\mathrm{m}}$ than fragment $\mathbf{1}$ and a number of compounds were identified and the affinities of these were assessed using ITC (Table 2 and Supplementary Figure 7). These compounds have the scaffold of Fragment 1 and an indole moiety connected with different linkers (Table 2). In addition, the crystal structures of MtDHFR:NADPH in complex with four of these compounds were obtained (Figure 4 and Supplementary Table 3). Interestingly the ITC indicated a significant increase of affinity for three molecules, in the range of 10, 20, and 40-fold greater than the initial fragment and the LE decreased only slightly for the compounds 1c and 1d, respectively (Table 2). The best of these compounds (1d) had a $K_{D}$ of $17 \mu \mathrm{M}$ and has a propylamine linker. The complexes of MtDHFR:NADPH with these 4 compounds had their crystallographic structures solved and the gain of the affinity of $\mathbf{1 d}$ was provided by the oxygen of the propanamide group linker that is able to form a new hydrogen 
bonding interaction with Gln28 side chain in contrast to the other compounds of this series (Figure 4). The analysis of the structures of the complexes indicates that probably a shorter linker is favorable to accommodating these compounds in the active site and performing $\pi$-interaction with Phe31. However, because of its flexibility, the linker can adopt different conformations as well as the indole ring, although this always performs a $\pi$ interaction with a Phe31. In contrast, the fluorine atom attached to the aryl ring of the compound 1a seems to strongly influence the affinity of this compound as it has the lowerest affinity amongst these series. The addition of the methyl group on the indole ring of compound 1c also could decrease the affinity of this compound in contrast to the 1d. The thermodynamic analysis of $\mathbf{1 d}$ indicates a high entropic penalty in comparison with the other compounds of the series (Supplementary Figure 7).

However, these four compounds have lost their interaction with the glycerol binding pocket adopting the binding mode of position 1, which it is shown in Figure 3A1, most likely due to the strong interaction between the indole group and Phe31, and the favourable interaction of the carboxylic acid with the Arg60 at the active site entrance. Further optimisation is necessary to maintain chemical functional groups in this pocket. 
bioRxiv preprint doi: https://doi.org/10.1101/2020.03.30.016204; this version posted April 1, 2020. The copyright holder for this preprint (which was not certified by peer review) is the author/funder, who has granted bioRxiv a license to display the preprint in perpetuity. It is made available under aCC-BY-NC-ND 4.0 International license.

Table 2. Calorimetric analysis of derivatives from Fragment 1.

\begin{tabular}{|c|c|c|c|}
\hline Compound & $\Delta \operatorname{Tm}\left({ }^{\circ} \mathrm{C}\right)$ & $\mathbf{K d}(\boldsymbol{\mu} \mathbf{M})$ & $\mathbf{L E}$ \\
\hline \multirow{2}{*}{ Frag 1} & 1.2 & $641 \pm 124$ & 0.28 \\
\hline & 3.3 & $192 \pm 84$ & 0.19 \\
\hline \multirow[t]{2}{*}{$1 a$} & & & \\
\hline & 3.3 & -- & --- \\
\hline \multicolumn{4}{|l|}{$1 b$} \\
\hline \multirow{2}{*}{$1 c$} & 3.6 & $34 \pm 8$ & 0.22 \\
\hline & 5.1 & $17 \pm 2$ & 0.24 \\
\hline $1 d$ & & & \\
\hline
\end{tabular}



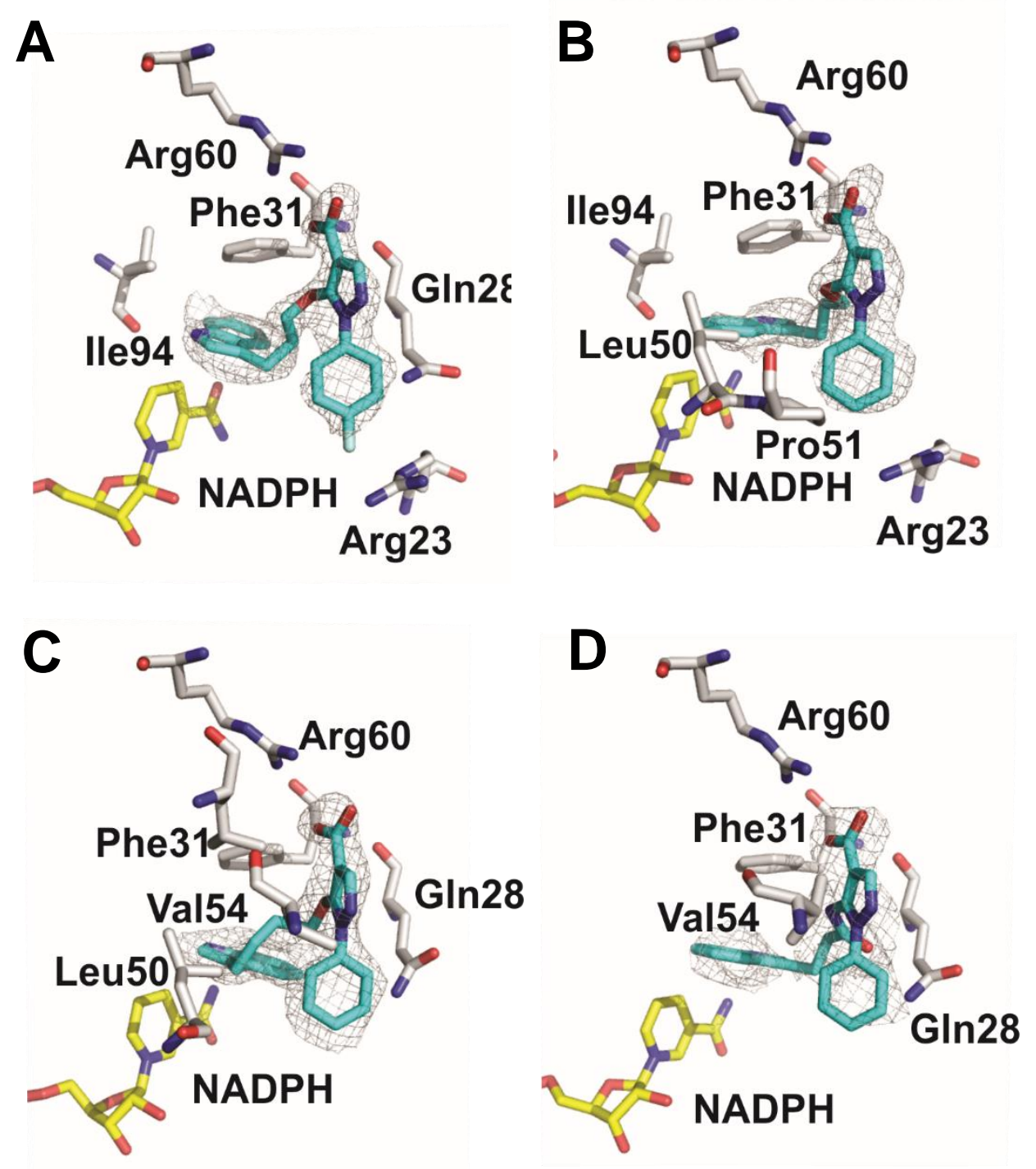

Figure 4. Crystallographic structures of compounds derived from Frag. 1. A) 1a (PDB entry 6VV6; B) 1b (PDB entry 6VV9); C) 1c (PDB entry 6VV7) D) 1d (PDB entry $6 \mathrm{VV} 8)$. The electron density is based on a $2 \mathrm{Fo}-\mathrm{Fc}$ map with a $1 \sigma$ contours.

The compounds based on fragment 1 show that the development of new DHFR inhibitors based on a pyrimidine free scaffold is feasible and a more stepwise approach for fragment growing, expanding into the rest of the active site whilst maintaining the glycerol binding site interaction should be possible. As the glycerol binding site is a specific feature of MtDHFR, selective compounds targeting M. tuberculosis rather than the human DHFR could be obtained. 


\section{Conclusions}

We have successfully applied an FBDD approach to the enzyme MtDHFR and have identified and confirmed the interactions of 21 molecules. This is the first report of an FBDD campaign against any DHFR and surprisingly, many of the identified compounds have completely novel scaffolds not reported for any DHFR. Through subsequent screening of an in-house library, derivatives of one of the molecules (1) were identified and binding assessed, showing improved affinities from $600 \mu \mathrm{M}$ to $17 \mu \mathrm{M}$, although interactions with the glycerol binding site were lost. However, the identification of these lead molecules opens possibilities in the identification of a completely novel series of compounds targeting MtDHFR and that could move forward to in vivo studies.

\section{Acknowledgments}

This research was supported by FAPESP (Sao Paulo Research Foundation, grants 2010/15971-3, 2014/09188-8 and 2018/00351-1, The Bill \& Melinda Gates Foundation (Grant RG48788 MAAG/555 and PHZF/121), CNPq grant 442021/2014-3 and Royal Society of Chemistry grant RF-17-9399. JAR and SMCP received fellowships from the FAPESP, fellowship 13/15906-5 and 17/25733-1, respectively. AH received support from DAAD, Cambridge Trust and Emmanuel College, Cambridge. GALZ received a fellowship from the Support Program for Foreign Ph.D. Student and Ibero-American Graduate Association (PAEDEx/AUIP 2014. TK receives an EPSRC Ph.D. fellowship and MVBD receives a CNPq research fellowship (Bolsa de Produtividade em Pesquisa, Level 2). We thank LNLS and LNBio at CNPEM in Campinas for allowing the use of the MX2 and NMR facilities, respectively; C. Oliveira and I. Cuccuvia from Institute of Physics and Institute of Chemistry, University of São Paulo, respectively for allowing the access of calorimetry facilities. 


\section{References}

1. Bertacine Dias, M. V, Santos, J. C., Libreros-Zúñiga, G. A., Ribeiro, J. A. \& Chavez-Pacheco, S. M. Folate biosynthesis pathway: mechanisms and insights into drug design for infectious diseases. Future medicinal chemistry 10, 935-959 (2018).

2. Gonen, N. \& Assaraf, Y. G. Antifolates in cancer therapy: Structure, activity and mechanisms of drug resistance. Drug Resistance Updates 15, 183-210 (2012).

3. Cipriani, P., Ruscitti, P., Carubbi, F., Liakouli, V. \& Giacomelli, R.

Methotrexate: an old new drug in autoimmune disease. Expert Rev Clin Immunol 10, 1519-1530 (2014).

4. Morgan, A., Cofer, C. \& Stevens, D. L. Iclaprim: a novel dihydrofolate reductase inhibitor for skin and soft tissue infections. Future Microbiology 4, 131-144 (2009).

5. Salcedo, E., Cortese, J. F., Plowe, C. V, Sims, P. F. \& Hyde, J. E. A bifunctional dihydrofolate synthetase--folylpolyglutamate synthetase in Plasmodium falciparum identified by functional complementation in yeast and bacteria. $\mathrm{Mol}$ Biochem Parasitol 112, 239-252 (2001).

6. Müller, I. B. \& Hyde, J. E. Folate metabolism in human malaria parasites - 75 years on. Molecular and Biochemical Parasitology 188, 63-77 (2013).

7. Sharma, M. \& Chauhan, P. M. Dihydrofolate reductase as a therapeutic target for infectious diseases: opportunities and challenges. Future Med Chem 4, 13351365 (2012). 
8. Sassetti, C. M., Boyd, D. H. \& Rubin, E. J. Comprehensive identification of conditionally essential genes in mycobacteria. Proc Natl Acad Sci U S A 98, 12712-12717 (2001).

9. Falcão, V. C. et al. Validation of Mycobacterium tuberculosis dihydroneopterin aldolase as a molecular target for anti-tuberculosis drug development. Biochem Biophys Res Commun 485, 814-819 (2017).

10. Yang, X. et al. 1,3,5-triazaspiro[5.5]undeca-2,4-dienes as selective Mycobacterium tuberculosis dihydrofolate reductase inhibitors with potent whole cell activity. European Journal of Medicinal Chemistry 144, 262-276 (2018).

11. Mugumbate, G. et al. Mycobacterial dihydrofolate reductase inhibitors identified using chemogenomic methods and in vitro validation. PloS one 10, e0121492 (2015).

12. Kumar, A. et al. High-throughput screening and sensitized bacteria identify an M. tuberculosis dihydrofolate reductase inhibitor with whole cell activity. PLoS One 7, e39961 (2012).

13. Vilchèze, C. \& Jacobs JR., W. R. Resistance to Isoniazid and Ethionamide in Mycobacterium tuberculosis: Genes, Mutations, and Causalities. Microbiology Spectrum 2, (2014).

14. Zheng, J. et al. para-Aminosalicylic acid is a prodrug targeting dihydrofolate reductase in Mycobacterium tuberculosis. J Biol Chem 288, 23447-23456 (2013).

15. Pai, M. et al. Tuberculosis. Nature Reviews Disease Primers (2016). 
doi:10.1038/nrdp.2016.76

16. Saunders, M. J. \& Evans, C. A. Fighting poverty to prevent tuberculosis. The Lancet Infectious Diseases 16, 395-396 (2016).

17. Barthod, L. et al. News on therapeutic management of MDR-tuberculosis: a literature review. Journal of Chemotherapy 30, 1-15 (2018).

18. Shuker, S. B., Hajduk, P. J., Meadows, R. P. \& Fesik, S. W. Discovering HighAffinity Ligands for Proteins: SAR by NMR. Science 274, 1531-1534 (1996).

19. Erlanson, D. A., Fesik, S. W., Hubbard, R. E., Jahnke, W. \& Jhoti, H. Twenty years on: the impact of fragments on drug discovery. Nature Reviews Drug Discovery 15, 605-619 (2016).

20. Romasanta, A. K. S. et al. When fragments link: a bibliometric perspective on the development of fragment-based drug discovery. Drug Discovery Today 23, 1596-1609 (2018).

21. Mashalidis, E. H., Śledź, P., Lang, S. \& Abell, C. A three-stage biophysical screening cascade for fragment-based drug discovery. Nature protocols $\mathbf{8}, 2309$ $24(2013)$.

22. Heikkila, T. J. et al. Fragment-based drug discovery in academia: Experiences from a tuberculosis programme. NATO Security through Science Series C: Environmental Security (2009). doi:10.1007/978-90-481-2339-1-3

23. Lamoree, B. \& Hubbard, R. E. Current perspectives in fragment-based lead discovery (FBLD). Essays in biochemistry 61, 453-464 (2017). 
24. Trapero, A. et al. Fragment-Based Approach to Targeting Inosine-5'-

monophosphate Dehydrogenase (IMPDH) from Mycobacterium tuberculosis.

Journal of Medicinal Chemistry 61, 2806-2822 (2018).

25. Collins, P. M. et al. Achieving a Good Crystal System for Crystallographic X-

Ray Fragment Screening. in Methods in enzymology 610, 251-264 (2018).

26. Hung, A. W. et al. Application of fragment growing and fragment linking to the discovery of inhibitors of Mycobacterium tuberculosis pantothenate synthetase. Angewandte Chemie (International ed. in English) 48, 8452-6 (2009).

27. Silvestre, H. L., Blundell, T. L., Abell, C. \& Ciulli, A. Integrated biophysical approach to fragment screening and validation for fragment-based lead discovery. Proc Natl Acad Sci U S A 110, 12984-12989 (2013).

28. Surade, S. et al. A structure-guided fragment-based approach for the discovery of allosteric inhibitors targeting the lipophilic binding site of transcription factor EthR. Biochemical Journal 458, 387-394 (2014).

29. Dai, R. et al. Fragment-Based Exploration of Binding Site Flexibility in Mycobacterium tuberculosis BioA. Journal of Medicinal Chemistry 58, 5208$5217(2015)$.

30. Marchetti, C., Chan, D. S. H., Coyne, A. G. \& Abell, C. Fragment-based approaches to TB drugs. Parasitology 145, 184-195 (2018).

31. Mendes, V. \& Blundell, T. L. Targeting tuberculosis using structure-guided fragment-based drug design. Drug Discov Today (2016).

doi:10.1016/j.drudis.2016.10.003 
32. Dias, M. V, Tyrakis, P., Domingues, R. R., Paes Leme, A. F. \& Blundell, T. L. Mycobacterium tuberculosis dihydrofolate reductase reveals two conformational states and a possible low affinity mechanism to antifolate drugs. Structure 22, 94-103 (2014).

33. Ribeiro, J. A. et al. Crystal structures of the closed form of Mycobacterium tuberculosis dihydrofolate reductase in complex with dihydrofolate and antifolates. Acta Crystallographica Section D Biol Crystallogr 75, 682-693 (2019).

34. Kabsch, W. XDS. Acta Crystallogr D Biol Crystallogr 66, 125-132 (2010).

35. Evans, P. R. \& Murshudov, G. N. How good are my data and what is the resolution? Acta Crystallogr D Biol Crystallogr 69, 1204-1214 (2013).

36. Winn, M. D. et al. Overview of the CCP4 suite and current developments. Acta Crystallogr D Biol Crystallogr 67, 235-242 (2011).

37. McCoy, A. J. et al. Phaser crystallographic software. J Appl Crystallogr 40, 658674 (2007).

38. Li, R. et al. Three-dimensional structure of M. tuberculosis dihydrofolate reductase reveals opportunities for the design of novel tuberculosis drugs. $J \mathrm{Mol}$ Biol 295, 307-323 (2000).

39. Adams, P. D. et al. The Phenix software for automated determination of macromolecular structures. Methods 55, 94-106 (2011).

40. Afonine, P. V et al. Towards automated crystallographic structure refinement with phenix.refine. Acta Crystallogr D Biol Crystallogr 68, 352-367 (2012). 
41. Emsley, P. \& Cowtan, K. Coot: model-building tools for molecular graphics. Acta Crystallogr D Biol Crystallogr 60, 2126-2132 (2004).

42. Chen, V. B. et al. MolProbity: all-atom structure validation for macromolecular crystallography. Acta Crystallogr D Biol Crystallogr 66, 12-21 (2010).

43. Congreve, M., Carr, R., Murray, C. \& Jhoti, H. A'rule of three'for fragmentbased lead discovery? Drug Discovery Today 8, 876 (2003).

44. Moreau, R. J. et al. Fragment-Based Drug Discovery of Inhibitors of Phosphopantetheine Adenylyltransferase from Gram-Negative Bacteria. Journal of Medicinal Chemistry 61, 3309-3324 (2018).

45. Srinivasan, B., Rodrigues, J. V., Tonddast-Navaei, S., Shakhnovich, E. \& Skolnick, J. Rational Design of Novel Allosteric Dihydrofolate Reductase Inhibitors Showing Antibacterial Effects on Drug-Resistant Escherichia coli Escape Variants. ACS Chemical Biology 12, 1848-1857 (2017).

46. Mayer, M. \& Meyer, B. Group Epitope Mapping by Saturation Transfer Difference NMR To Identify Segments of a Ligand in Direct Contact with a Protein Receptor. (2001). doi:10.1021/ja0100120

47. Sugiki, T., Furuita, K., Fujiwara, T. \& Kojima, C. Current NMR Techniques for Structure-Based Drug Discovery. Molecules 23, 148 (2018). 